\title{
A Linear Transform for Spectral Estimation
}

\author{
MIGUEL A. LAGUNAS-HERNÁNDEZ, MEMBER, IEEE, ANÍBAL R. FIGUEIRAS-VIDAL, MEMBER, IEEE, \\ JOSÉ B. MARIÑO-ACEBAL, AND ANTONIO CAROL VILANOVA
}

\begin{abstract}
The authors propose the introduction of a previous linear transform for rational spectral estimation by using a linear predictor. The method is based on works of Oppenheim [1], [2] on discrete signal representation.

The connection with zero-pole (ARMA) spectral estimators is discussed, and the main potential advantages of the approach are indicated: reduction in estimator order, low implementation complexity, and the possibility of increasing frequency resolution.

The use of this known linear transform seems to be useful in cases in which the presence of a strong periodicity in the signal to be analyzed is a priori known.

Some promising results obtained in processing signals from hydrographic measurements are shown.
\end{abstract}

\section{INTRODUCTION}

A LINEAR predictor of length $Q$ for a sample of a stochastic process $\{x\}$ is of the form

$$
\hat{x}(k)=\sum_{i=1}^{Q} a(i) \cdot x(k-i)
$$

in which the process is assumed to be zero mean and stationary. If the coefficients $\{a(i)\}$ are appropriately calculated, $\hat{x}(k)$ will approximate the sample $x(k)$ in a certain sense. The usual criterion consists of minimizing the mean square of the prediction error

$$
e(k)=x(k)-\hat{x}(k),
$$

i.e.,

$$
E\left[e^{2}(k)\right]=E\left\{\left[x(k)-\sum_{i=1}^{Q} a(i) x(k-i)\right]^{2}\right\} .
$$

The coefficients $\{a(i)\}$ obtained by minimizing (3) are the solutions of the normal system

$$
\sum_{i=1}^{Q} a(i) R_{x x}(l-i)=R_{x x}(l), \quad l=1, \cdots, Q
$$

where $R_{x x}(\cdot)$ is the autocorrelation function of the process.

A spectral estimator for $\{x\}$ can be derived from this linear predictor (see [3], for example). It is predicated on the fact that for sufficiently large $Q,\{e(k)\}$ will typically converge to a white process with covariance $\sigma^{2}$.

Then the resulting spectral estimator is

$$
\hat{S}_{x x}(\omega)=\sigma^{2} /\left|1-\sum_{k=1}^{Q} a(k) \exp (-j k \omega \tau)\right|^{2}
$$

Manuscript received August 6, 1980; revised January 6, 1981.

M. A. Lagunas-Hernández and A. Carol Vilanova are with ETSI Telecomunicación, Jorge Girona Salgado s/n, Barcelona, Spain.

A. R. Figueiras-Vidal and J. B. Mariño-Acebal are with ETSI Telecomunicación, Cdad. Universitaria, Madrid, Spain. where $\tau$ is the sampling period assumed for the samples $x(n)$.

This kind of rational spectral estimator, which implicates an AR model for $\{x\}$, is at the present time one of the most useful and practical procedures for spectral estimation. Interesting and related methods are Burg's maximum entropy (MEM) [4], [5] and Widrow's adaptive methods [6], [7].

Despite its apparent attractiveness, the spectral form (5) has drawbacks. The estimator does not have zeros. This limitation is so remarkable that Welch's method [8], based on the periodogram, shows better results in some cases than does MEM [with moving average (MA) sequences]. The absence of zeros also creates problems when the signal to be processed is contaminated by a high noise component [9].

There exist some techniques which include zeros in the previous estimator and which would alleviate these drawbacks to some degree. They include generalizations of Widrow's algorithm [10], the ITIF of Konvalinka and Matausek [11], Steiglitz's method [12], and the method of Gutowski et al. [13]. Many of these require a high degree of computational effort.

In the present work, we suggest the application of a linear transform to the available data as an indirect method for including zeros. This will often allow us to reduce the order of the all-pole (AR) estimator on the transformed data. The resulting method needs prior knowledge about the existence of a main peak in the power spectrum, or, in other words, if a main periodicity in the measured samples is present. The approximate duration of the mentioned periodicity is also needed in order to design the final power spectrum estimator.

It must be noticed that the presentation here is oriented to parametric methods in power spectral estimation, but, in some sense, the involved ideas can be applied in nonparametric methods like the Blackman-Tukey method and Welch's method.

We use the transformation in parametric methods because, in our personal point of view, they are well suited to take advantage of local or global characteristics of the signal to be analyzed in order to include some kind of preprocessing or to modify the estimator structure to obtain good quality spectral estimators.

In fact, windowing and overlapping techniques can be viewed as previous data transformations (we refer the reader to references [14] and [15] for a good discussion about these particular preprocessing nonparametric methods). But, including modifications derived trom previous knowledge about the problem under analysis, it is usually more difficult in these methods than in parametric ones.

Of course, a parametric method which uses specific characteristics of the signal $x(n)$ does not have a wide application 


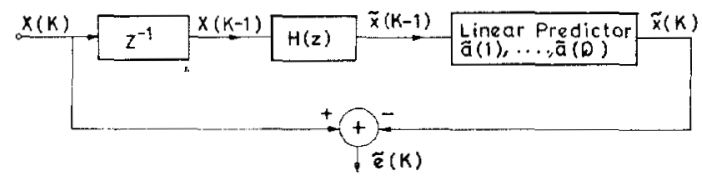

Fig. 1. One-step predictor with data preprocessing.

and, clearly, a tradeoff between its performance for particular signals and its usefulness in general problems exists.

We recall that the features of the transformation described in this paper apply only to the cases where a main periodicity in the signal $x(n)$ exists. If it does not exist, general transformations, such as Parcor formulation [16] or orthonormal transforms [17] in parametric methods, and data or correlation windowing and overlapping in nonparametric methods will exhibit better results than the transformation proposed here.

The resulting algorithm, compatible with any adaptation, seems to reduce the order of the estimator to obtain comparable results. It does not implicate hard computational effort, and, additionally, it allows an increase in resolution obtained from the incorporated transform.

We will show an example with an adaptive algorithm, applied on original and on transformed data, for comparison purposes.

\section{A Previous Transform for Linear Prediction}

If we introduce the matrix notation

$$
\begin{aligned}
X_{k-1} & =[x(k-1) \cdots x(k-Q)] \\
A^{t} & =[a(1) \cdots a(Q)] \\
P^{t} & =\left[R_{x x}(1) \cdots R_{x x}(Q)\right] \\
R & =\left[\begin{array}{cccc}
R_{x x}(0) & R_{x x}(1) & \cdots & R_{x x}(Q-1) \\
R_{x x}(1) & R_{x x}(0) & \cdots & R_{x x}(Q-2) \\
\vdots & & & \vdots \\
R_{x x}(Q-1) & R_{x x}(Q-2) & \cdots & R_{x x}(0)
\end{array}\right]
\end{aligned}
$$

(where $t$ indicates transposition), then (1), (2), and (4) can be written in the form

$$
\begin{aligned}
\hat{x}(k) & =X_{k-1} A=x(k)-e(k) \\
R A & =P .
\end{aligned}
$$

If we apply preprocessing on the data $X_{k-1}$, we will obtain a new data vector $\widetilde{X}_{k-1}$ (see Fig. 1) to predict $x(k)$. The optimum coefficients $\tilde{A}$, which minimize the covariance of the new error $\tilde{e}(k)$, can be obtained from the matrix $\widetilde{R}$ and the matrix $\widetilde{P}$ which are derived from $\widetilde{X}_{k-1}$ in the same way as (8) and (9) are derived from (6). Then (12) and (13) become

$$
\begin{aligned}
\tilde{x}(k) & =\widetilde{X}_{k-1} \tilde{A}=x(k)-\tilde{e}(k) \\
\tilde{R} \tilde{A} & =\widetilde{P}
\end{aligned}
$$

where $\tilde{x}(k)$ is the new predicted value. Assuming that the preprocessing is linear and time-invariant, characterized by a transfer function $H(z)$, then the new estimate of $S_{x x}(\omega)$ associated with the new predictor will be (Fig. 1)

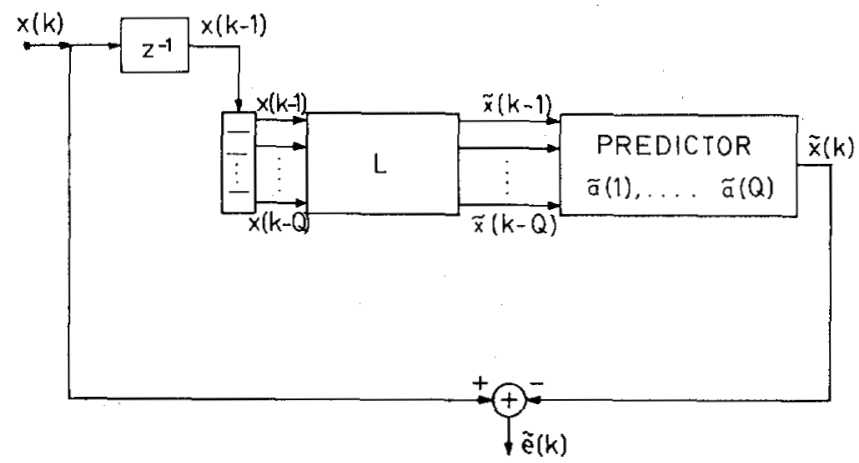

Fig. 2. Predictor with a previous linear transform and the corresponding prediction error.

$$
\tilde{S}_{x x}(\omega)=\tilde{\sigma}^{2} /\left|1-\sum_{k=1}^{Q} \tilde{a}(k) \exp (-j k \omega \tau) H[\exp (j \omega \tau)]\right|^{2}
$$

where $\tilde{\sigma}^{2}$ is the covariance of $\widetilde{e}(k)$. When $H(z)$ corresponds to a recursive filter (IIR), its poles become the zeros of the spectral estimator. The above formula shows the relation between ARMA models and previous preprocessing.

The inherent drawback of this indirect method for including zeros with respect to direct methods is that some previous knowledge of the structure of the data is required in order to decide what kind of preprocessing is adequate.

To gain more insight about the mentioned preprocessing, in Section III we will obtain some guidelines to decide what kind of data preprocessing is adequate. Also, we will return to the general problem of a linear transformation characterized by a transition matrix $L$ as we can see in (15) (Fig. 2):

$$
\tilde{X}_{k-1}^{t}=L X_{k-1}^{t} \text {. }
$$

It is clear that when $L$ is a Toeplitz matrix, we can characterize the transformation using a linear system $H(z)$ with initial zero conditions. But our interest is centered in the general transformation denoted by (15).

\section{EfFect of the Transformation on the Order of the Predictor}

It is not difficult to obtain formulas (16a) and (16b) from the previous equations:

$$
\begin{aligned}
& \sigma^{2}=E\left[e^{2}(k)\right]=R_{x x}(0)-P^{t} R^{-1} P \\
& \tilde{\sigma}^{2}=E\left[\tilde{e}^{2}(k)\right]=R_{x x}(0)-\tilde{P}^{t} \tilde{R}^{-1} \tilde{P} .
\end{aligned}
$$

From (15)

$$
\widetilde{P}=E\left[x(k) \widetilde{X}_{k-1}^{t}\right]=L P
$$

and, if $L^{-1}$ exists

$$
|\widetilde{R}|^{-1}=\left[E\left(\tilde{X}_{k-1}^{t} \widetilde{X}_{k-1}\right)\right]^{-1}=\left(L^{t}\right)^{-1} R^{-1}(L)^{-1} .
$$

Inserting (17a) and (17b) in (16b), it is verified that both mean-square errors are equal. This can be also viewed in Fig. 3 , in a case of a second-order predictor, and $L$ corresponding to a rotation.

Thus, it is clear that a linear transform does not reduce the mean square error. In other words, since the transform leads new data in the same hyperplane defined by the old data, the 


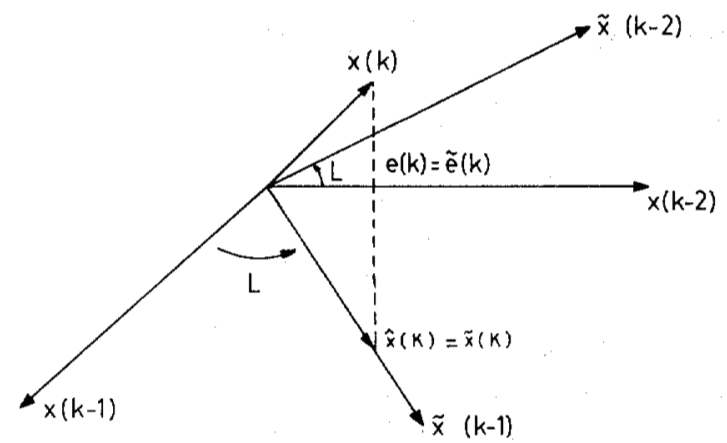

Fig. 3. A transform applied on geometrical representation for a secondorder one-step predictor.

minimum distance from $\hat{x}(k)$ or $\widetilde{x}(k)$ to $x(k)$ (sample to be predicted) remains. However, from Fig. 3 , one can infer that if $L$ leads a sample to the position of the $x(k)$ projection on the data plane, the order of the predictor will be reduced to one. Generalizing it, if a transform or preprocessing leads to such a data set that a part of them is more correlated with the sample to be predicted $x(k)$, and the other part of data less correlated, the first part length will be enough to obtain a new predictor with (approximately) the same mean-square error. Then, we must search for a transform such that $E[x(k) \tilde{x}(k-$ $m)]$ be appreciable for values of $m$ as low as possible.

An additional practical consideration is of importance. If $\tilde{R}$ is nearly diagonal, its inversion will be straightforward, and the computational time per iteration in adaptive methods will be decreased.

Thus, we must select $L$ according to the following requisites.

1) Obtaining data maximally correlated with $x(k)$ and forcing a nearly diagonal matrix $\widetilde{R}$ for the new data.

2) Low complexity, so as not to lose the computational advantages derived from the above properties.

\section{The Proposed Transform}

Assuming now that a main periodicity is present in the signal under analysis [e.g., 12 months in hydrographic data, $100 \mathrm{~ms}$ in EEG (electroencephalographic) registers, pitch period in voiced speech, etc.], we propose, following the guidelines mentioned in Section III, the insertion of a delay $T_{0}$ among the data used to predict $x(k)$. The value $T_{0}$ should be approximately the duration of the mentioned periodicity.

$$
T_{0}=M \cdot \tau \text {. }
$$

This assumption corresponds to admit relative maxima each $M$ points in $R_{x x}(m)$; then we expect higher correlation among the ensemble $x(k-i \cdot M)(i=1, Q)$ and $x(k)$ than among the ensemble $x(k-i)(i=1, Q)$ and $x(k)$. Fig. 4 shows an example of an autocorrelation function derived from a simulated signal with a periodicity $M \tau$ and also shows how a one-step predictor works in both cases: delay $\tau$ and delay $M \tau$ among data.

Fig. 5 indicates the structure of the modified predictor, assuming that $H(z)$ introduces the desired delay $M \tau$. The predictor error $\widetilde{e}(k)$ would be used to update the $\{\widetilde{a}(i)\}$ in an adaptive algorithm.

To clarify the involved idea we can give the following example. In a meteorological problem, the periodic structure of the data, using data obtained from the same month in

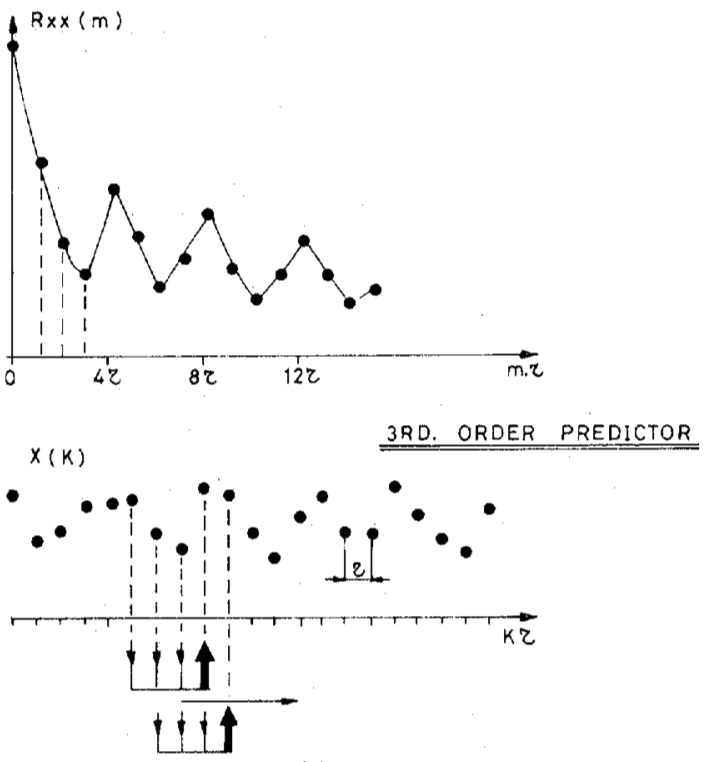

(a)

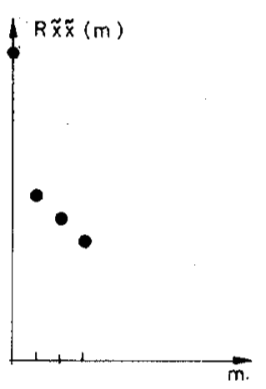

3RD. ORDER PREDICTOR

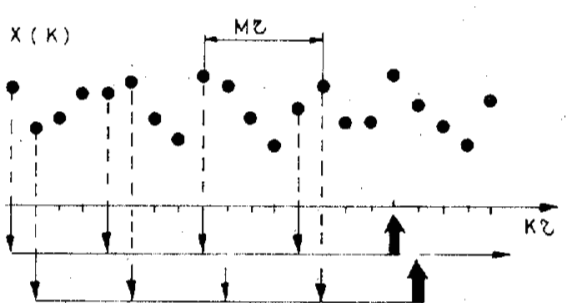

(b)

Fig. 4. Data correlation and predictor scheme for: (a) Delay $\tau$ between samples. (b) Delay $M \tau$ between samples.

different years, provides better prediction than using previous months' data in the current year.

We select a delay filter having only a pole

$$
H(z)=\frac{\alpha-z^{-1}}{1-\alpha z^{-1}}
$$

where $\alpha$ is forced to introduce the desired delay in the region of the spectra where the main periodicity is centered. This approximation of a delay $M \tau$ at all frequencies allows a memory reduction with respect to a nonrecursive filter with a transfer function $z^{-M}$.

From Fig. 5 the new estimator will be

$$
\tilde{S}_{x x}(\omega)=\tilde{\sigma}^{2} /\left|1-\sum_{k=1}^{Q} \tilde{a}(k)\{H[\exp (j \omega \tau)]\}^{k}\right|^{2} .
$$




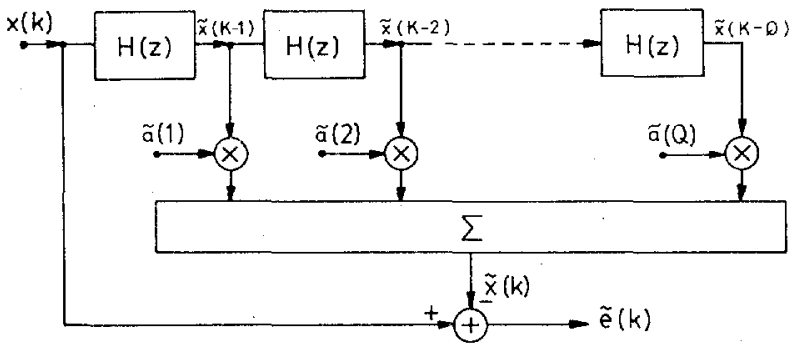

Fig. 5. The proposed predictor using $T_{0}$ recursive delays.

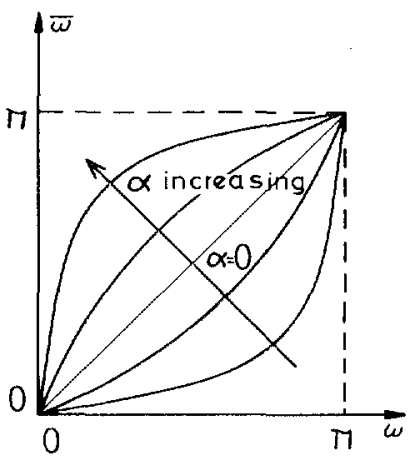

Fig. 6. Frequency compression/expansion by means of a recursive filter with only a pole.

Based on the all-pass character of the filter $H(z)$, we can introduce a new (angular) frequency $\tilde{\omega}$, defined by (21a) and $(21 \mathrm{~b})$

$$
\begin{aligned}
\tilde{z} & =H(z) \\
\exp (j \tilde{\omega} \tau) & =H(\exp (j \omega \tau)) .
\end{aligned}
$$

Then (20) becomes

$$
\tilde{S}_{x x}(\tilde{\omega})=\tilde{\sigma}^{2} /\left|1-\sum_{k=1}^{Q} \tilde{a}(k) \exp (j k \tilde{\omega} \tau)\right|^{2} .
$$

To introduce the (angular) frequency $\tilde{\omega}$ is equivalent to a new scaling of the frequency axis; this new scaling is shown in (23) assuming a unit sampling period $\tau$.

$$
\tilde{\omega}=\tan ^{-1}\left\{\left(1-\alpha^{2}\right) \sin \omega /\left[\left(1+\alpha^{2}\right) \cos \omega-2 \alpha\right]\right\} .
$$

This has been already considered by Oppenheim [1], [2] .

Fig. 6 illustrates how different values of $\alpha$ implicate different compression/expansion of frequency regions. This magnifying effect of low frequencies can be translated to high or intermediate frequencies by selecting another filter to implement the delay $M \tau$.

This magnifying effect must not be considered as a simple increase of resolution like selective linear prediction methods [3], which use frequency warping techniques. We remark that our interest is in the use of new data more correlated with the sample to be predicted and the warping effect was a secondary result. In fact, this work shows that a modified predictor, like that shown in Fig. 5, implicates a proper choice for the all-pass filters $H(z)$ if a quality improvement of the estimate is desired.

\section{Some Results}

To illustrate the application of the previous ideas, we will apply our procedure in a hydrographic problem.

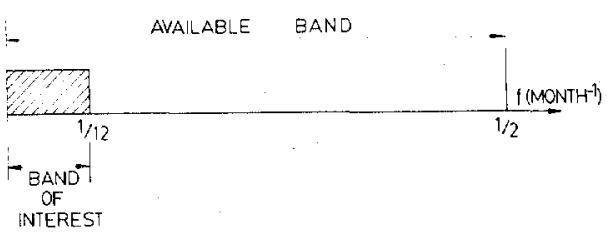

Fig. 7. Available band and band of interest in the example, without transform.

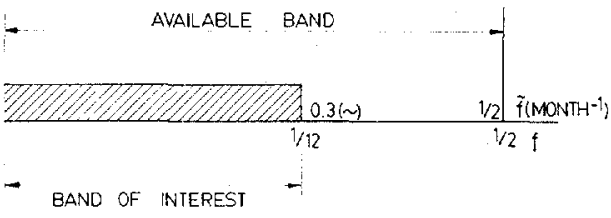

Fig. 8. Available band and band of interest in the example, transforming with $\alpha=0.7$.

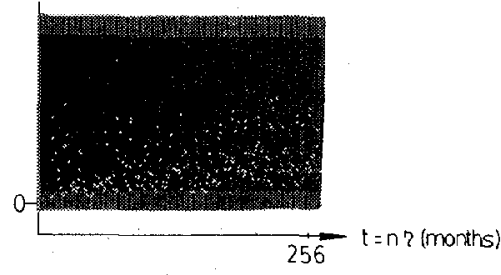

Fig. 9. A river flow sampled monthly.

In hydrographic and many other meteorological signals, long periodicities are of great interest. These periodicities are located at frequencies lower than the main yearly peak of the spectra. When $\tau$ represents a month, we must use a delay $T_{0}=$ $12 \tau$ (twelve months) in the design of the modified predictor.

Then, the magnifying effect previously discussed is useful in these cases: without transforming, the zone of interest would be $\frac{1}{6}$ of the available frequency band (Fig. 7); with filter $H(z)$ of twelve months delay at a frequency $\frac{1}{12}$ months $^{-1}$, which is required to improve the quality of the estimator, the zone of interest is extended in the form shown in Fig. 8 (approximately).

Fig. 9 is a river flow sampled 256 times within a monthly period. Fig. 10 includes spectral estimations obtained without and with transforming [(a) and (b), respectively] by means of Griffiths's algorithm [7] and order 15, where the coefficients at iteration $k+1$ are

$$
\tilde{a}^{(k+1)}(i)=\tilde{a}^{(k)}(i)+\mu \widetilde{x}(k-i) \widetilde{e}(k)
$$

and $\mu$ is a convergence parameter.

The improvement is evident: two periodicities, which, from a specialists' opinion, are really present in the river flow's behavior, one every four years and another (slight) every eleven years. These periodicities are detected with the transform and they are not detected without it. Predictors having orders 100 and 120 , respectively, have been necessary for detecting these periodicities without using the transform. We have obtained similar results with simulated signals, and we have observed that slight deviations of $\alpha$ around its correct value introduce false peaks and, at the same time, decrease the values of true peaks.

These and other experiences carried out by the authors have shown a reduction of at least 20 percent in the predictor order for equivalent estimations working with meteorological signals; with other kinds of signals this reduction varies depending on the relative importance of the main 


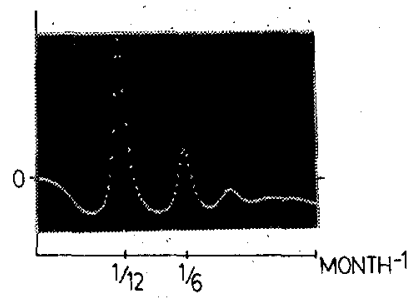

(a)

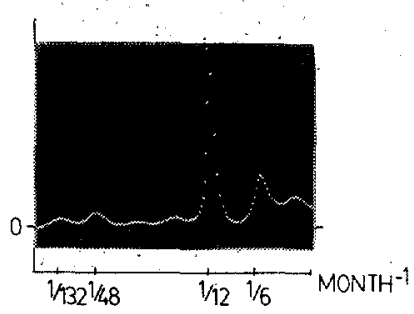

(b)

Fig. 10. (a) Spectral estimation of the river flow without transform. (b) With transform. The same algorithm and order (Griffiths, 15) are used in both cases.

periodicity. In EEG analysis, 15 percent seems to be the available reduction.

\section{CONCLUSION}

The use of an adequately selected transform of the available data allows a reduction in the order of the spectral estimation necessary to obtain a prescribed detail. This results in a reduction of the computational complexity as well as in introducing zeros in the spectral estimator.

In the case of signals with a main (strong) known periodicity corresponding to $M$ times the sampling interval $\tau$, a delay $M \tau$ takes the hole of the linear transform. Implementing (approximately) this delay by means of an all-pass recursive filter reduces the required memory and magnifies the frequency band. At the same time we obtain an improvement in the estimator quality as a main result. This is of special interest when long periodicities are significant as it occurs with meteorological and hydrographic signals. Other kinds of recursive filters can be applied to magnify other frequency bands.

The method seems to be useful, apart from spectral estimations in the indicated cases, to detect a transient and instantaneous frequency using adaptive algorithms.

The disadvantage of the proposed technique consists of requiring a previous knowledge of the problem for selecting an adequate transform; but, in many cases, the designers will have this knowledge.

\section{ACKNOWLEDGMENT}

The authors are grateful to Dr. M. Bertrán-Salvans for providing data and for his helpful comments, and to the reviewers of the original manuscript for their useful suggestions.

\section{REFERENCES}

[1] A. V. Oppenheim and D. H. Johnson, "Discrete representation of signals," Proc. IEEE, vol. 60, pp. 681-691, June 1972.

[2] A. V. Oppenheim, D. H. Johnson, and K. Steiglitz, "Computation of spectra with unequal resolution using the fast Fourier transform," Proc. IEEE, vol. 59, pp. 283-297, June 1975.

[3] J. Makhoul, "Spectral linear prediction: Properties and applications," IEEE Trans. Acoust., Speech, Signal Processing, vol. ASSP-23, pp. 283-297, June 1975.
[4] J. P. Burg, "Maximum entropy spectral analysis," in Proc. 37th Meeting Soc. Expl. Geophysicists, Oklahoma City, OK, Oct. 1967.

[5] T. J. Ulrych and T. N. Bishop, "Maximum entropy spectral analysis and autoregressive decomposition," Rev. Geophys. Space Phys., vol. 13, pp. 183-200, Feb. 1975.

[6] B. Widrow et al., "Stationary and nonstationary learning characteristics of the LMS adaptive filter," Proc. IEEE, vol. 64, pp. 1151-1162, Aug. 1976.

[7] L. J. Griffiths, "Rapid measurement of digital instantaneous frequency," IEEE Trans. Acoust., Speech, Signal Processing, vol. ASSP-23, pp. 207-222, Apr. 1975.

[8] P. D. Welch, "The use of fast Fourier transform for the estimation of power spectra: A method based on time averaging over short modified periodograms," IEEE Trans. Audio Electroacoust., vol. AU-15, pp. 70-73, June 1967.

[9] S. M. Kay, "The effects of noise on the autoregressive spectral estimator," IEEE Trans. Acoust., Speech, Signal Processing, vol. ASSP-27, pp. 478-485, Oct. 1979.

[10] B. Widrow and J. M. McCool, "Comments on an adaptive recursive LMS filter," Proc. IEEE, vol. 65, pp. 1402-1404, Sept. 1977.

[11] J. S. Konvalinka and M. R. Matausek, "Simultaneous estimation of poles and zeros in speech analysis and ITIF-Iterative inverse filtering algorithm," IEEE Trans. Acoust., Speech, Signal Processing, vol. ASSP-27, pp. 492-501, Oct. 1979.

[12] K. Steiglitz, "On the simultaneous estimation of poles and zeros in speech analysis," IEEE Trans. Acoust., Speech, Signal Processing, vol. ASSP-25, pp. 229-234, June 1977.

[13] P. R. Gutowski, E. A. Robinson, and S. Treitel, "Spectral estimation: Fact or fiction," IEEE Trans. Geosci. Electron., vol. GE-16, pp. 80-84, Apr. 1978.

[14] A. H. Nuttall and G. C. Carter, "A generalized framework for power spectral estimation," IEEE Trans. Acoust., Speech, Signal Processing, vol. ASSP-28, pp. 334-335, June 1980.

[15] C. K. Yuen, "Comments on modern methods for spectrum estimation," IEEE Trans. Acoust., Speech, Signal Processing, vol. ASSP-25, pp. 298-299, June 1979.

[16] J. Makhoul, "Stable and efficient lattice methods for linear prediction," IEEE Trans. Acoust., Speech, Signal Processing, vol. ASSP-25, pp. 423-428, Oct. 1977.

[17] M. A. Alam, "Adaptive spectral estimation," in Proc. Joint Automat. Contr. Conf., vol. 1, pp. 105-112, June 1977.

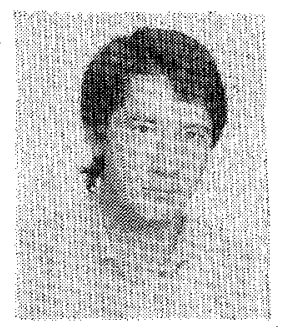

Miguel A. Lagunas-Hernández (S'73-M'78) was born in Madrid, Spain, on October 11, 1951. $\mathrm{He}$ received the Telecommunication Engineer degree from the ETSI Telecomunicación, Universidad Politécnica de Madrid; Madrid, in 1973 , and the Doctor degree in telecommunication engineering from the ETSI Telecomunicación, Universidad Politécnica de Barcelona, Barcelona, Spain, in 1976.

After his graduation he became Professor of Electronics and Signal Processing at ETSI Telecomunicación, Barcelona. His research activities have been centered on signal processing, especially on spectral analysis, and clinical, meteorological, and speech applications. He has been a member of the organizing committees of several international conferences.

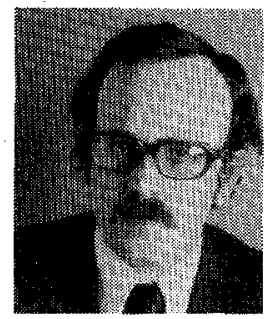

Aníbal R. Figueiras-Vidal (S'74-M'76) was born in Vigo, Spain, on May 6,1950 . He received the Telecommunication Engineer degree from the ETSI Telecomunicación, Universidad Politécnica de Madrid, Madrid, Spain, in 1973, receiving a national award at graduation and the Doctor degree (Honors) in telecommunication engineering from the ETSI Telecomunicación, Universidad Politécnica de Barcelona, Barcelona, Spain, in 1976

From 1973 until 1978 he was an Assistant and an Associate Professor at ETSI Telecomunicación, Barcelona. Since 1978, he has been a Professor at ETSI Telecomunicación, Madrid, where he is Vice-Head at the present time. He has held several fellowships and received several distinctions during his academic life. His re 
search interests are in communications theory and systems and in signal processing.

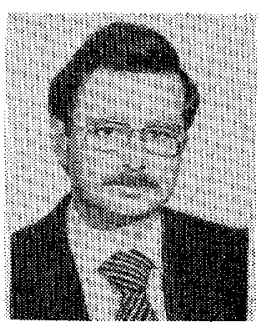

José B. Mariño-Acebal was born in La Coruña, Spain, on March 19, 1950. He received the Telecommunication Engineer degree from the ETSI Telecomunicación, Universidad Politécnica de Madrid, Madrid, Spain, in 1973, and the Doctor degree in telecommunication engineering from the ETSI Telecomunicación, Universidad Politécnica de Barcelona, Barcelona, Spain, in 1975.

From 1972 until 1980 he was Professor of network theory at ETSI Telecomunicación,
Barcelona. In 1980 he joined ETSI Telecomunicación, Madrid. His areas of interest are signal processing and network theory.

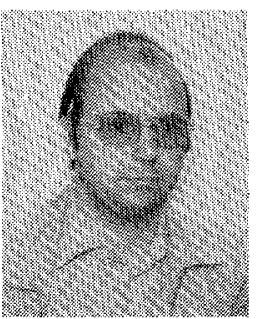

Antonio Carol Vilanova was born in Barcelona, Spain, in 1953. He received the Telecommunication Engineer degree from the ETSI Telecomunicación, Universidad Politécnica de Barcelona, Barcelona, Spain, in 1978.

He is pursuing the Doctor degree in telecommunication engineering at the Universidad Politécnica de Barcelona, where he is currently Assistant Professor. His work is in the area of digital signal processing with emphasis on biomedical applications.

\title{
Sampling the 2-D Radon Transform
}

\author{
PAUL A. RATTEY, STUDENT MEMBER, IEEE, AND ALLEN G. LINDGREN, MEMbER, IEEE
}

\begin{abstract}
The Radon transform of a bivariate function, which has application in tomographic imaging, has traditionally been viewed as a parametrized univariate function. In this paper, the Radon transform is instead viewed as a bivariate function and two-dimensional sampling theory is used to address sampling and information content issues. It is shown that the band region of the Radon transform of a function with a finite space-bandwidth product is a "finite-length bowtie." Because of the special shape of this band region. "Nyquist sampling" of the Radon transform is on a hexagonal grid. This sampling grid requires approximately one-half as many samples as the rectangular grid obtained from the traditional viewpoint. It is also shown that for a nonbandlimited function of finite spatial support, the bandregion of the Radon transform is an "infinite-length bowtie." Consequently, it follows that approximately $2 M^{2} / \pi$ independent pieces of information about the function can be extracted from $M$ "projections." These results and others follow very naturally from the two-dimensional viewpoint presented.
\end{abstract}

\section{INTRODUCTION}

$\mathrm{T}$ HE Radon transform of a function on $\mathbb{R}^{2}$ arises naturally in the tomographic imaging problem which is concerned with reconstructing a cross section of an object from measurements which are integrals of the "cross-section function" over lines. Each line-integral measurement is a sample of the Radon transform of the cross-section function. If a sufficient set of these Radon transform samples is known, then the Radon transform is adequately specified and the cross-section func-

Manuscript received June 25, 1980. This work was supported by the National Science Foundation under Grant ENG 77-20927.

The authors are with the Department of Electrical Engineering, University of Rhode Island, Kingston, RI 02881. tion can be determined by inverting the Radon transform. Typical examples of cross-section functions include the attenuation function in transmission computer-assisted tomography [1], the refractive-index function in ultrasonic refractive-index tomography [2], the reflectivity function in ultrasonic reflectivity tomography [3], the radio-emission function in radio astronomy [4], and the molecular-structure function in electron microscopy [5]. An X-ray transmission tomography example is illustrated in Fig. 1(a). In that figure, a three-dimensional object (the head) is decomposed into a set of parallel slices. In this way, a three-dimensional reconstruction problem can be viewed as a set of two-dimensional reconstruction problems.

This article focuses on: 1) the sampling requirements which must be imposed on the Radon transform in order that it be uniquely determined; and 2) the implications of sampling the Radon transform with a limited number of "views" or "projections." The sampling requirements are developed with a two-dimensional viewpoint and contrasted with the requirements developed from the usual one-dimensional viewpoint [5], [6]. By imposing standard approximations, it is shown that the bandregion of the Radon transform of a "bandlimited" function of finite spatial support has the shape of a "finite-length bowtie." Because of this special bandregion, it follows that "Nyquist" sampling of the Radon transform must be on a hexagonal grid. This sampling grid requires approximately one-half as many samples as the rectangular grid obtained from the traditional viewpoint. This result clarifies apparent discrepancies that exist in the literature regarding 